\title{
UNA POLÍTICA PARA EL DESARROLLO DE LA MICRO, PEQUEÑA Y MEDIANA EMPRESA EN PASTO (COLOMBIA)
}

\section{A POLICY FOR THE DEVELOPMENT OF MICRO, SMALL AND MEDIUM ENTERPRISES IN PASTO (COLOMBIA)}

\author{
Edwin Tarapuez Chamorro ${ }^{1}$
}

Fecha de recibido: Febrero 3 de 2010

Fecha de aceptado: Junio 9 de 2010

Correspondencia: Programa de Contaduría Pública. Universidad del Quindío Av. Bolivar calle 12 norte Armenia Quindío. Correo electrónico: edwintarapuez@yahoo.es

\section{RESUMEN}

El proyecto de investigación denominado "Estudio para el diseño de una política productiva encaminada hacia el desarrollo, promoción y fortalecimiento de la micro, pequeña y mediana empresa (MIPYME) en Pasto", fue financiado por la Institución Universitaria CESMAG, a través de la Vicerrectoría de Investigaciones, dentro de la linea de investigación de Desarrollo Empresarial. Dicho proyecto tuvo como objetivo general, concertar y diseñar la propuesta de una política para el desarrollo, promoción y fortalecimiento de la MIPYME en dicho municipio. Contó con el apoyo de entidades públicas y privadas que directamente influyen en el desarrollo de las empresas del municipio y que participaron a través de reuniones, conversatorios y diligenciamiento de encuestas, en la definición de los elementos constitutivos de la política de desarrollo para la MIPYME. Después de sistematizar y priorizar la información obtenida se concluyó que la política municipal de desarrollo de la MIPYME debe ser liderada por la Alcaldía Municipal y debe estar orientada por los principios de integralidad, cooperación, flexibilidad y universalidad.

De otra parte el trabajo de investigación permitió priorizar, con los participantes, cinco campos de acción en los que los actores involucrados deben aportar sus esfuerzos en forma coherente y colectiva. Dichos campos son: ambiental, capacitación del talento humano, comercialización, financiamiento e impuestos municipales. La conclusión más importante del trabajo es que la coordinación interinstitucional del gobierno local con el sector privado, la academia y otras entidades gubernamentales es posiblemente la variable clave para desarrollar con éxito una política para el desarrollo de la MIPYME a nivel local. El documento final contiene elementos útiles para cualquier municipio de latinoamérica, dada su problemática común.

Palabras clave: Municipio, Desarrollo Empresarial, Desarrollo Económico, Desarrollo Regional, PYME, MIPYME, MYPE

\begin{abstract}
The research project entitled "Study for the design of a productive policy aimed to development, promotion and strengthening of micro, small and medium enterprises (MSMEs) in Pasto" was financed by Research Subdirection of Institucion Universitaria CESMAG within the research line of Business Development. The main objectives of this project were to concert, and design the proposal of a policy to develop, promotion and strengthen of MSME in the municipality of Pasto. The project had the support of public and private entities directly related with entrepreneurial development in the municipality and their participation was through meetings, talks and filling out surveys in the definition of the constituent elements of the development policy for MSMEs. After systematized and prioritized the obtained information it was concluded that the municipally policy of develop of SME should be led by the Mayor's office and must be guided by principles of integrity, cooperation, flexibility and universality. This research work allowed along with the participants, five fields of action in which people should contribute their efforts in a coherent and collective way. These fields are: environmental, human talent training, marketing, finance and taxes. The most important finding of this study is that coordination between government institutions and the private sector, academic environment and other government agencies is probably the key for successful development of a policy for the development of local MSMEs. The final document contains elements useful for any municipality in Latin America, given their common problems.
\end{abstract}

Keywords: municipality, Business Development, Economic Development, Regional Development, MSME

${ }^{1}$ Docente Programa de Contaduria Universidad del Quindío. 


\section{INTRODUCCIÓN}

El tema del desarrollo regional es relativamente nuevo y ha generado mucho interés entre los diferentes actores sociales que han aportado distintos enfoques y propuestas a través de los cuales se ha tratado de explicar y orientar la evolución de las regiones.

En las décadas de los treinta y los cuarenta se pensó que la configuración del mercado nacional haría posible el desarrollo armónico de las regiones de los países de América Latina. De igual manera en los años cincuenta y sesenta se pensaba que el comercio internacional, con base en una nueva división del trabajo por sustitución de importaciones, resolvería las crecientes diferencias del desarrollo tanto entre las naciones como entre las regiones de los países ${ }^{1}$.

Lo cierto es que en los últimos años se ha puesto de manifiesto que en los mercados nacionales conviven regiones superdesarrolladas e infradesarrolladas. En este sentido, el centralismo trajo como consecuencia el favorecimiento de zonas particulares, como ciudades capitales, las áreas metropolitanas que representaban una posición financiera e industrial crucial, o las que tendrían un papel estratégico en el proceso de unificación nacional. De ahí que se afirme comúnmente que las cabezas de centralización financiera e industrial son los factores de origen del subdesarrollo regional ${ }^{2}$.

El presente documento se basa en los resultados del proyecto de investigación denominado "Estudio para el diseño de una política productiva encaminada hacia el desarrollo, promoción y fortalecimiento de la micro, pequeña y mediana empresa (MIPYME) en Pasto", que fue financiado por la Institución Universitaria CESMAG, a través de la Vicerrectoría de Investigaciones, dentro de la linea de investigación de Desarrollo Empresarial. Su objetivo general fue concertar y diseñar la propuesta de una política para el desarrollo, promoción y fortalecimiento de la MIPYME en Pasto.

\section{Metodología}

\subsection{Tipo de estudio}

El estudio realizado fue de carácter cualitativo y exploratorio.

Algunas de las variables que se tuvo en cuenta fueron: o Instrumentos legales a nivel local para el desarrollo de la MIPYME.

o Instrumentos legales a nivel nacional para el desarrollo de la MIPYME.

- El papel de los gremios de la producción y demás entidades públicas y privadas en el desarrollo de la MIPYME local.

o Principios orientadores y elementos constitutivos para una política integral de desarrollo de la MIPYME.

\subsection{Universo y muestra}

Para el desarrollo de la investigación se procedió a realizar una sensibilización con los representantes de las entidades y empresas que se habían vinculado al proyecto, con el fin de dar a conocer el proyecto, los alcances y objetivos.

Las entidades vinculadas fueron:

\subsection{Instrumentos de recolección de información}

La investigación obtuvo información primaria de los directivos y representantes de las entidades y empresas mencionadas anteriormente, a través de reuniones, conversatorios y encuestas realizadas durante un periodo de 8 meses.

De igual manera se recurrió a fuentes secundarias como:

o Documentos y estadísticas de Cámara de Comercio de Pasto, FENALCO Seccional Nariño, ACOPI Seccional Nariño, Contactar, Fondo Regional de Garantías de Nariño S.A, SENA, Concejo Municipal de Pasto, Asamblea Departamental de Nariño, Alcaldía de Pasto y Gobernación de Nariño.

- Libros, artículos de revistas, prensa e informes de entidades nacionales.

o Tesis de grado relacionadas con el sector productivo local.

\section{Resultados}

Los resultados obtenidos del proyecto se centraron en:

- La concertación sobre los cuatro principios básicos que debe contener una política de desarrollo para la micro, pequeña y mediana empresa en el municipio.

\footnotetext{
${ }^{1}$ Un análisis complementario se puede consultar en: León, N. (1997). "Conceptos, estrategias y gestión del desarrollo local”. En: VARIOS AUTORES. Análisis regional y empleo. Un enfoque multidisciplinario (17-62). Santafé de Bogotá : Ministerio de Trabajo-Universidad Nacional-CID.

${ }_{2}^{2}$ Molina, I. (1997). Nueva regionalización mundial, desarrollo local y gobernabilidad. En Revista Comercio Exterior, 47(11), p. 935-936.
} 
- La priorización de los cinco componentes básicos para la implementación de una política local para el desarrollo de la MIPYME en el municipio de Pasto.

o Definición de las principales acciones a seguir en cada uno de los componentes de la política de desarrollo de la MIPYME.

Dichos resultados se tratan en forma más detallada a continuación.

\subsection{Política municipal para el desarrollo de las MIPYMES}

Una política municipal para el desarrollo de la MIPYME busca generar un apoyo integral a estas empresas, de tal forma que se eviten los esfuerzos aislados de los diferentes agentes del municipio y que dichos esfuerzos se realicen sobre las distintas variables que influyen en el desarrollo empresarial.

En este mismo sentido busca desarrollar el potencial de las personas y grupos sociales del municipio en la medida en que el gobierno local debe facilitar los mecanismos para acercarse a uno de los anhelos más arraigados en las personas cual es el de ser dueños de su propia empresa, es decir, promover el emprendimiento.

\subsection{Justificación}

El tema de la creación de empresas se convirtió en política nacional con la aprobación de la Ley 590 de 2000, más conocida como Ley MIPYME, que posteriormente fue ajustada a través de la ley 905 de 2004. Por ello, y con el fin de apoyar la política nacional, es necesario que a nivel municipal se genere y adopte una política local para el desarrollo de la micro, pequeña y mediana empresa, que apoye en el ámbito regional la aplicación de los instrumentos de carácter nacional y que a la vez promueva la aplicación y diseño de otros instrumentos que a nivel territorial son de su competencia.

En las reuniones realizadas los asistentes afirmaron, casi que en consenso, que en el municipio no existe cultura empresarial. Ello es totalmente discutible, pues todos los municipios poseen una cultura empresarial, sino que cada una es diferente a la de las demás regiones del país y del mundo.

De otra parte, ante el cambio de modelo económico, la crisis de la economía colombiana (y por ende de las regionales) y los consecuentes recortes de personal de las empresas públicas y privadas, se hace necesario diseñar una política municipal que promueva el desarrollo de la MIPYME como un mecanismo ideal para la generación de empleo, redistribución de la riqueza, generación de mayor valor agregado y mayores recursos para inversión pública por la vía impositiva. Este mecanismo se viene implementando a nivel mundial como consecuencia de una tendencia global.

De igual manera, así como el Estado pretende estimular al sector privado para generar más y mejores empresas, debe paralelamente crear los instrumentos necesarios para que este proceso sea mejor y más expedito, que minimice los obstáculos institucionales que dificultan la creación y desarrollo de las empresas y que a la vez les brinde un apoyo integral.

\subsection{Principios orientadores de la política productiva}

En términos generales los representantes de las entidades vinculadas al estudio coincidieron en que la política productiva para el desarrollo de la MIPYME debe tener unos principios orientadores que definan la filosofía de la misma. Estos principios identificados por los actores fueron: integralidad, cooperación, flexibilidad y universalidad.

\section{Integralidad}

Tradicionalmente los instrumentos de apoyo al sector de las MIPYMES han estado enfocados a brindar soporte en los diferentes campos del desarrollo empresarial. De esta manera, se han establecido programas de capacitación, mercadeo y comercialización, exenciones tributarias y asistencia técnica, entre otros.

Estos programas han adolecido de la integralidad necesaria que le permita a las empresas aprovechar cabalmente los diferentes programas y estrategias de apoyo.

Con este principio se pretende evitar la implementación de programas aislados que conlleven a resultados parciales que no desarrollen la empresa como un todo, es decir, se busca que en el desarrollo de la política se actúe en los distintos campos y variables que influyen en el desarrollo empresarial.

\section{Cooperación}

En muchas regiones del país se sostiene que a la gente no le gusta unirse para crear empresa. Este factor se presenta en mayor o menor medida en todas las regiones del país y en todos los países del mundo. La individualidad, la desconfianza y el anhelo de poseer algo propio, son características propias de la naturaleza humana que se desarrollan más en unas culturas que en otras dependiendo de ciertos factores que pueden alentarlas a crecery desarrollarse.

Rev. Invest. Univ. Quindío (20): 99 - 107. Armenia - Colombia 
Con este principio se busca aunar esfuerzos entre todos los actores regionales que influyen en el desarrollo de la MIPYME.

\section{Flexibilidad}

Los distintos grados de desarrollo de las empresas, un entorno socioeconómico relativamente cambiante, al igual que las diferentes posibilidades, capacidades, habilidades y destrezas de los actuales y futuros empresarios, demandan flexibilidad en los programas y estrategias que se adopten dentro de una política municipal para el desarrollo de las MIPYMES.

Con este principio se busca que la política tenga la facultad para moldearse con relativa facilidad ante los cambios del entorno globalizado para que pueda responder de manera oportuna ante dichos cambios.

\section{Universalidad}

Una política municipal para el desarrollo empresarial se enfrenta a una variada gama de empresas en los diferentes sectores económicos, con distintos tamaños y diferente clasificación. Se enfrenta, igualmente, a diferentes concepciones religiosas, políticas e ideológicas por parte de los empresarios o candidatos a serlo.
Por ello, una política municipal para el desarrollo empresarial no debe discriminar el tamaño ni el sector económico de las empresas, ni la condición social, religiosa, sexual o política de su (s) propietario (s), ni su procedencia regional.

Con este principio se busca que todas las empresas puedan formar parte de la política municipal para el desarrollo de la MIPYME, sin dejar de lado la importancia de buscar sectores estratégicos en los que el municipio es o puede ser competitivo.

\subsection{Principales componentes de una política para el desarrollo de las MIPYMES}

Una política para el desarrollo de las MIPYMES debe ser integral y sincronizar los esfuerzos del gobierno local, gremios de la producción, empresarios, universidades y demás entidades vinculadas con este sector.

En este sentido, se busca que las empresas sean el centro de todos los intentos por mejorar el desarrollo empresarial de la región.

Esta sincronización de esfuerzos se puede mostrar con un esquema circular de esfuerzos como el que se muestra en la Figura 1.

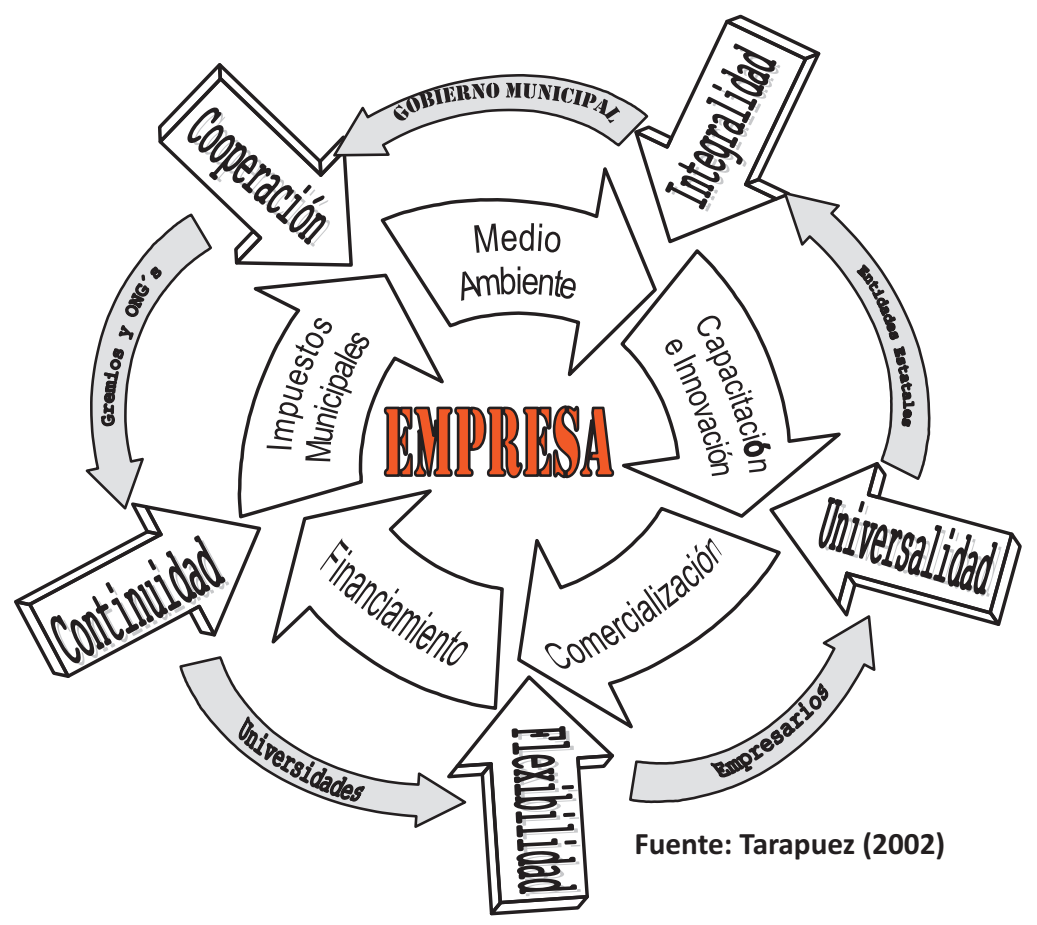

Figura 1. Esquema circular de esfuerzos en el diseño de una política municipal para el desarrollo de la micro, pequeña y mediana empresa 
Hablar de una política municipal de desarrollo productivo implica tener muy presente, además de los principios de integralidad, cooperación, flexibilidad y universalidad, mencionados en anteriores apartes, el desarrollo de actividades estratégicas ordenadas y lideradas por la administración municipal en los campos ambiental, de capacitación del talento humano, comercialización, financiamiento e impuestos municipales, sin perder de vista que la coordinación interinstitucional con el sector privado y otras entidades descentralizadas del gobierno nacional es especialmente importante para mejorar la coherencia de los programas y acciones.

\subsubsection{Política Ambiental}

En un contexto global como el que se vive en la actualidad, es fundamental que se garantice un desarrollo empresarial que promueva la utilización de tecnologías no contaminantes de tal forma que se asegure la sostenibilidad del medio ambiente.

En el proceso de formulación de las políticas ambientales del Municipio debe darse una interacción directa entre el gobierno local y las empresas ubicadas en su jurisdicción.

Tradicionalmente los instrumentos de gestión ambiental se han clasificado en la literatura entre la intervención directa y el control y los incentivos económicos. Con relación a los primeros se requiere de un aparato institucional de vigilancia del cumplimiento importante, y generan pocos incentivos para el cambio tecnológico y cultural hacia prácticas de producción y consumo más "limpias" o sostenibles. Los incentivos, por su diseño más flexible a la racionalidad de los agentes económicos, pueden requerir menos costos de implementación o vigilancia, pero en algunos casos información técnica más costosa para su diseño.

\section{Acciones generales en la política ambiental}

- Determinar algunas exenciones tributarias a las empresas que se reubiquen en zonas de menor impacto ambiental, que reemplacen su maquinaria y equipos por otros menos contaminantes, que reduzcan su impacto ambiental negativo y que utilicen materiales de reciclaje dentro de su proceso de transformación, entre otros.

- Gran parte de los empresarios miran las restricciones y normas ambientales como una gran amenaza que eleva los costos de producción y operación de sus empresas. Por ello es necesario realizar campañas de concientización sobre el medio ambiente.

- Es necesario replantear la necesidad de la actualización de maquinaria y equipo contaminante por otras tecnologías que generen un menor impacto ambiental negativo.

- La labor de las Secretarías Municipales del Medio Ambiente y de las Corporaciones Autónomas Regionales debe ser de acompañamiento y educación al empresario y no simplemente de un control punitivo sobre las fallas estructurales de la economía local que se reflejan en las MIPYMES.

- La reubicación de algunas empresas debe estar acompañada de medidas alternativas para evitar el desplazamiento per se de dichas empresas.

- La creación de Parques Industriales es una acción prioritaria, no solamente para reducir el impacto ambiental de las industrias locales sino para generar economías de escala en la región.

- Las entidades competentes deben permitir que las empresas del mismo subsector puedan realizar estudios de impacto ambiental de manera asociada ya que gran parte de las causas y efectos tiene un mismo origen.

- En general los empresarios piensan que la contaminación solamente se da por vertimiento de residuos a las fuentes de agua y de gases a la atmósfera. Ello requiere el desarrollo de un programa de capacitación y educación al sector productivo para dar a conocer la gran variedad de formas de contaminación.

\subsubsection{Política de capacitación del talento humano}

Podría afirmarse que una auténtica democracia implica necesariamente una política de desarrollo del talento humano, es decir, que en la medida que las regiones, los municipios y el país en general cuenten con oportunidades de capacitación a todo nivel y de acuerdo a los requerimientos del sector productivo, se generará una distribución más equitativa de oportunidades laborales, económicas y sociales para los habitantes de la región.

Ante todo se hace necesario estructurar una política municipal orientada a potenciar la capacidad empresarial y de gestión, así como el recurso humano de las MIPYMES.

De otra parte se habla en el país del divorcio entre la universidad y el sector productivo. En realidad puede afirmarse que sí existe una relación aunque en muchos casos no está dada de manera muy formal ni continua.

A pesar de ello debe recalcarse que faltan canales de comunicación que retroalimenten a ambas partes sobre las necesidades de capacitación de las empresas y la calidad de la educación y formación que se imparte en las aulas universitarias.

Lo anterior no significa que dicha relación no deba fortalecerse y estructurarse mucho más. De hecho muchas universidades viven pendientes solamente de superar a sus competidores independientemente de la calidad que ofrecen y de su relación con el sector empresarial. A la vez, muchos 
empresarios viven encerrados en su diario quehacer de alcances cortoplacistas.

En cuanto al desarrollo tecnológico (a pesar de que no se propone una política exclusiva para esta variable), es conveniente una acción pública de promoción de empresas, organizaciones y redes universitario-tecnológicasempresariales en las que se acumule y circule el conocimiento sobre las formas organizativas y tecnológicas que permitan un aumento de la productividad no basado exclusivamente en el aumento de capital fijo sino en el aumento de nuevos proyectos de base tecnológica ${ }^{3}$.

\section{Acciones generales en la política de talento humano}

- Estimular a los empresarios a adquirir servicios de capacitación y asesoría para prepararse con mayor proyección en el nuevo entorno de la economía global.

- Creación de programas de educación formal y no formal enfocados a la capacitación gerencial y oferta de cursos y seminarios a nivel de todas las facultades de las universidades y centros de educación superior.

- Inclusión de la metodología de educación empresarial en las instituciones de educación superior locales y en los colegios del municipio, para la promoción de empresarios líderes y el desarrollo del espíritu empresarial. Debe revisarse los pénsums académicos de todas las carreras y no solamente las del perfil empresarial (un avance, aunque no definitivo, es la inclusión de asignaturas como Creatividad Empresarial y Desarrollo de la mentalidad emprendedora en algunas universidades de la región).

- Mayor vinculación de los directivos universitarios con el medio externo.

- Creación y desarrollo de muestras empresariales con los proyectos productivos más innovadores que creen los estudiantes de colegio y universidades.

- Mejoramiento de los programas de capacitación y asistencia técnica que brinda el Servicio Nacional de Aprendizaje (SENA).

- Desarrollo de programas conjuntos y complementarios de capacitación y asistencia técnica en las empresas.

- Generar programas básicos de cualificación que vinculen a los empresarios con los computadores e internet, ya que estas son dos herramientas muy valiosas para el desarrollo de las MIPYMES que aún no se han establecido en muchas empresas.

- Generar beneficios tributarios a las empresas que capaciten a sus trabajadores o vinculen laboralmente a personas de los sectores más vulnerables de la población (minusválidos, jóvenes, mujeres y desplazados, entre otros)

- Desarrollo de programas para la formación de asesores y consultores especializados en temas de MIPYME.

- Gestión de programas de cooperación internacional para la vinculación de expertos jubilados de otros países, con amplia experiencia en asesoría y consultoría en empresas MIPYMES.

- Articulación de esfuerzos con las diferentes instituciones públicas y privadas que realizan cualificación empresarial Sería conveniente especializar la capacitación por entidades o sectores económicos y que se genere un plan anual de cualificación empresarial para que se evite repetir algunos programas.

\subsubsection{Política de Comercialización}

Los problemas de comercialización que afectan a las MIPYMES se enfocan básicamente a la tendencia local del mercado de las empresas ubicadas en el municipio y a la falta de información de mercado para una adecuada toma de decisiones estratégicas.

Aunque la mayor parte de las MIPYMES orientan sus ventas hacia el mercado doméstico, se considera indispensable iniciar una política cuyo objetivo sea el de modificar la visión de los negocios. En este sentido es necesario que el gobierno municipal y las demás entidades encargadas de la promoción empresarial, promuevan la mentalización del empresariado en torno a que debe emplearse una gran variedad de estrategias que motiven el consumo de los productos locales y se evite la conquista del mercado local por parte de productos importados de otros departamentos o países.

\section{Acciones generales en la política de comercialización}

- Generar una red de proveedores del gobierno municipal que se encargue de ofrecer los bienes y servicios que demanda la Alcaldía.

- Generación de espacios periódicos de promoción comercial (preferiblemente especializados), tales como muestras comerciales, ruedas de negocios y ferias especializadas.

- Capacitación en comercialización, atención al cliente, participación en eventos comerciales, estrategias de mercadeo y comercio internacional, entre otros temas.

- Adopción, por parte del gobierno municipal y de todos sus establecimientos, de un estatuto de contratación que promueva la vinculación de las empresas y recursos locales. Dicho estatuto debe estar en concordancia con

\footnotetext{
$\overline{3}$ Ver un análisis más amplio en: Waissbluth, M. (1998). El financiamiento gubernamental a la innovación. En Revista Comercio Exterior, $48(7), 547$ - 561.
} 
la Ley 80 de 1993. Un ejemplo valioso es el Estatuto de Contratación para las Empresas de Servicios Públicos Domiciliarios de Cali.

- Generar paulatinamente procesos de producción ambientalmente sostenibles para exportación de "productos ecológicos".

- Vincular a las universidades en la sensibilización y capacitación de los empresarios para la utilización de internet como herramienta de para promover el comercio electrónico.

- Vincular a los estudiantes de los últimos semestres de ingeniería y tecnología en sistemas de las universidades regionales en la asesoría para el diseño de las páginas web de las empresas locales.

- Determinar algunas exenciones tributarias para las empresas que sobrepasen cierto límite de ventas anuales.

- Vincular a entidades como el Laboratorio Colombiano de diseño en nuevos programas que mejoren la presentación de los productos locales.

- Gestionar los recursos necesarios para lograr los certificados de calidad necesarios para poder competir en mercados internacionales con productos manufactorados (ISO 9000, ISO 14000 y Sello Ecológico, entre otros).

- Generar mayor impacto en los programas de Proexport y Zeiky desarrollados en la región.

- Promover la participación de empresarios locales en misiones comerciales a otros países.

\subsubsection{Política de Financiamiento}

Tradicionalmente el sector financiero ha desempeñado un papel determinante en la promoción del desarrollo económico. Con la globalización, la apertura económica y el crecimiento de la competencia, este sector se ha convertido en clave para aumentar la inversión y la productividad y las condiciones necesarias de inserción en los mercados internacionales.

Esta es una de las variables en la que más énfasis hace el empresario ya que normalmente el las MIPYMES no disponen de efectivo para capital de trabajo, para adquisición de maquinaria o para ampliación de su producción. Por ello es de tanto interés para los empresarios del sector.

\section{Acciones generales en la política de financiamiento}

- Creación de una entidad de crédito para los empresarios del municipio.

- A nivel municipal entidades como las Cámaras de
Comercio, Cajas de Compensación Familiar y Fondos Regionales de Garantías, entre otras, disponen o podrían disponer de recursos o estrategias de soporte de crédito para creación de empresas. Estas entidades podrían unirse para otorgar planes conjuntos de financiación al sector de las MIPYMES municipales.

- La Alcaldía Municipal, conjuntamente con otras instituciones privadas y los empresarios podrían constituir un Fondo Regional de Capital de Riesgo encargado de financiar aquéllos proyectos de empresa viables financiera y estratégicamente de acuerdo con los estudios pertinentes (por ejemplo, un Estudio de Competitividad del municipio). Temporalmente el Fondo sería socio de las empresas que se apoye.

- Exenciones tributarias o incentivos a las entidades financieras que intermedien recursos de crédito como Bancoldex y Finagro hacia las MIPYMES locales.

- Generar sinergia entre el gobierno municipal, instituciones locales (incubadoras de empresas, centros de desarrollo tecnológico, unidades de emprendimento) y entidades del orden nacional como Colciencias y Sena (Fondo Emprender y Recursos de Ley 344) para obtener recursos no reembolsables de capital semilla para nuevos proyectos en el municipio.

\subsubsection{Política de impuestos municipales}

La variable impuestos es tal vez una de las más difíciles de controlar por parte del gobierno con respecto al recaudo que se genera de acuerdo con la actividad productiva de una determinada zona.

En últimas, cuando se tiene que exigir el pago de impuestos de una manera general y compulsiva, se observa una gran debilidad de la Alcaldía para satisfacer las necesidades de los contribuyentes del Municipio. Es fundamental, dentro de la gestión municipal, que la Alcaldía recompense con creces los impuestos que pagan sus contribuyentes, ya que a nadie le gusta participar en una relación donde una de las partes cumple con su obligación sin recibir la contraprestación por lo que está cancelando.

A través de una política de impuestos municipales se podrían articular gran parte de los elementos de las demás políticas propuestas.

\section{Posibles tipos de exenciones}

A través de una política de impuestos municipales se podrían articular gran parte de los elementos de las demás políticas, dependiendo de las condiciones locales: 
1. Exenciones por establecimiento de nuevas empresas dentro del territorio municipal y/o por ampliación productiva

2. Exenciones por generación de puestos de trabajo adicionales.

3. Exenciones por reubicación de empresas en determinadas zonas del municipio

4. Exenciones por reconversión industrial, desarrollo tecnológico o actualización de maquinaria y equipos

5. Exenciones por utilización de materiales de reciclaje en el proceso productivo o participación en campañas ambientales, entre otras.

6. Exenciones por formalización de vendedores ambulantes y estacionarios

7. Exenciones por capacitación en temas prioritarios para el desarrollo empresarial.

8. Exenciones por vinculación de personas discapacitadas en calidad de empleados o de los grupos de personas que presentan los mayores niveles de desempleo (jóvenes, mujeres, profesionales de determinada edad, etc.)

9. Exenciones a empresas intensivas en mano de obra

10. Exenciones a empresas que sobrepasen cierto límite de ventas anuales o que exporten, entre otras.

11. Exenciones a empresas que se encuentren asociadas con otras

12. Exenciones a instituciones financieras por intermediación de recursos de crédito para MIPYMES

A pesar de lo anotado inicialmente en este aparte, la variable impuestos puede constituirse en un mecanismo favorable para la ampliación, creación, fortalecimiento y desarrollo de las MIPYMES del municipio. Con esto sólo se pretende afirmar que las exenciones tributarias pueden ser eventualmente un mecanismo para promover la creación, fortalecimiento y desarrollo de nuevas empresas en el territorio. Es una variable sobre la que los representantes del gobierno municipal tienen bastante aversión para tramitar exenciones tributarias.

\subsection{Ente articulador de la política}

La implementación de una política municipal para el desarrollo de las MIPYMES requiere que los empresarios tengan un interlocutor directo en el gobierno municipal, es decir que debe existir un encargado con el cual éstos se relacionen. Para llevar a cabo este propósito, se pueden dar dos modalidades:

1. A través de la redefinición de funciones de alguna dependencia municipal, o

2. Creación de una Secretaría u oficina municipal para tal propósito
La implementación de cualquier alternativa dependerá de las condiciones de cada municipio. El desarrollo que alcance el programa no dependerá tanto de su ubicación dentro de la estructura municipal, sino principalmente de la inversión que se realice en talento humano especializado, equipamiento, infraestructura y gastos de funcionamiento ${ }^{4}$.

\section{Conclusiones}

A tono con las nuevas orientaciones internacionales, el desarrollo local y regional viene cobrando cada vez una mayor importancia como consecuencia de la falta de resultados positivos dentro de los diferentes modelos de desarrollo centralizado que habían venido imponiéndose en todo el mundo.

En este sentido, ante el cambio de modelo económico, el Municipio se erige como la célula más importante del territorio nacional, no en cuanto a extensión sino en lo referente a todos los procesos socioeconómicos y culturales que en el interior de cada localidad se llevan a cabo.

Hablar de una política municipal de desarrollo productivo implica tener muy presente los principios de integralidad, cooperación, flexibilidad y universalidad, que deben ser desarrollados por la Alcaldía Municipal para la implementación de un esfuerzo coherente y colectivo en los campos ambiental, de capacitación del talento humano, comercialización, financiamiento e impuestos municipales, sin perder de vista que la coordinación interinstitucional con el sector privado y otras entidades descentralizadas del gobierno nacional es especialmente importante para mejorar la coherencia de los programas y acciones.

Gracias al trabajo que han venido desarrollando en la actualidad algunas instituciones como la Agencia de Desarrollo Local y la Corporación Incubadora de Empresas de Nariño (fundadas conjuntamente por 17 entidades del ámbito regional), entidades que aún no se habían creado cuando se realizó la investigación, se ha logrado articular esfuerzos comunes no solamente para su conformación sino también en la gestión de iniciativas de impacto regional que de una u otra manera se han cristalizado en proyectos de interés regional para la promoción conjunta del desarrollo empresarial.

Gracias a una conciencia de los últimos gobernantes, tanto a nivel del departamento de Nariño como de su capital, Pasto, se han logrado incluir iniciativas y rubros presupuestales en los planes de desarrollo para promover un mayor nivel de

\footnotetext{
${ }^{4}$ Asociación chilena de municipalidades, op. cit. p. 93-94.
} 
competitividad de las MIPYMES de la región, exenciones tributarias para las nuevas empresas, gestión de recursos para creación de empresas y programas de capacitación enfocados a la promoción de proyectos de base tecnológica, entre otros. Estos esfuerzos, aunque aún no son suficientes, han permitido generar un clima de mayor confianza por parte del sector empresarial del municipio.
Quizá una de las condiciones imprescindibles para la implementación de una política para el desarrollo de la MIPYMES es contar con la voluntad política de los actores involucrados en el proceso. En la actualidad esa voluntad ha creado sinergia en la región, sin querer decir que no existen conflictos. Sin este componente sería muy difícil articular acciones interinstitucionales y enfocar los esfuerzos hacia un horizonte común.

\section{BIBLIOGRAFÍA}

Acopi Nariño (1999). Diagnóstico sectorial de la PMI nariñense y plan de acción. 1991-1992. Pasto: la asociación. (mimeo).

Acopi Nariño (1998). Programa de desarrollo empresarial sectorial PRODES sector de molinería: diagnóstico y plan de acción, 1998. Pasto; la asociación. (mimeo).

Asociación chilena de municipalidades (1995). El desarrollo económico local. Santiago de Chile : FESCOL

Boisier, S. (1996). La modernización del Estado: una mirada desde las regiones. Revoluciones, reformas, objetivos nacionales y el papel del territorio. En: Medina, J. y Varela, E. (Compiladores). Globalización y gestión del desarrollo regional. Cali : Editorial Universidad del Valle.

Cabrera, C. y otros (1996). Diagnóstico y perspectivas del desarrollo del subsector de artes gráficas en el municipio de Pasto. Pasto. Trabajo de Grado (Administrador Financiero). Centro de Estudios Superiores María Goretti. Facultad de Ciencias Administrativas.

Cabrera, H. (1997) Nuestro sistema financiero regional. En: Pasto Hoy: órgano de difusión de la Cámara de Comercio de Pasto. Edición XII (sep.).p. 8-11.

Cámara de Comercio de Pasto (1996). Análisis de las actividades comerciales, industriales y económicas del municipio de Pasto. En: Pasto Hoy : órgano de difusión de la Cámara de Comercio de Pasto. Edición X (sep.). p. 18-24.

Cámara de Comercio de Pasto (1998). Estudio de Competitividad y Perfiles de Proyectos de Inversión para el Departamento de Nariño. Síntesis. Pasto: la entidad.

Cámara de Comercio de Pasto (2000a). Estudio del comercio informal en la ciudad de San Juan de Pasto. Pasto: la entidad (mimeo).

Cámara de Comercio de Pasto (2000b). Huella regional de Nariño e identificación de clústers. Pasto : la entidad.

Cámara de Comercio de Pasto (2004). Censo Empresarial y Comercial 2003 en el Municipio de Pasto. Pasto: la entidad.

Gonzalez, P. (1998). La política industrial en la era de la globalización. En Revista Comercio Exterior, 48(7), 532 - 540.

León, N. (1997). "Conceptos, estrategias y gestión del desarrollo local”. En: VARIOS AUTORES. Análisis regional y empleo. Un enfoque multidisciplinario (17-62). Santafé de Bogotá : Ministerio de Trabajo-Universidad Nacional-CID.

Gobernacion de Nariño (2004). Plan Estratégico de Desarrollo de Nariño. 2004-2007. Pasto: la entidad.

Molina, I. (1997). Nueva regionalización mundial, desarrollo local y gobernabilidad. En Revista Comercio Exterior, 47(11), 929 - 942.

Pinto, J. y Suarez, F. (1999). Política de talento humano para la Pyme en Colombia. En: VARIOS AUTORES. Desarrollo de la pequeña y mediana empresa en Colombia (113-139). Santafé de Bogotá : BID-DNP-ACOPI.

Ramirez, M. (1997). Crecimiento o Desarrollo en América Latina: una historia en construcción. En Revista Comercio Exterior, 47(6), 473 479.

Rangel, A. (1993). El desarrollo regional. Santafé de Bogotá : ESAP.

Sili, M. (1997). La gestión empresarial en el desarrollo regional de América Latina. En Revista Comercio Exterior, 47(6), 460 - 465.

Tarapuez, E. (2002) Forjando un municipio empresarial. Pasto, Institución Universitaria CESMAG.

Tarapuez, E. (1999). Municipio y desarrollo empresarial. En Tiempos Nuevos: Revista institucional Centro de Estudios Superiores María Goretti. Año 4, No. 6 (julio). p. 3-5.

Vinueza, G. (1996) Sistemas de garantías para la micro, pequeña y mediana empresa. En Pasto Hoy : órgano de difusión de la Cámara de Comercio de Pasto. Edición X (sep.). p. 9-11.

Waissbluth, M. (1998). El financiamiento gubernamental a la innovación. En Revista Comercio Exterior, 48(7), 547 - 561. 